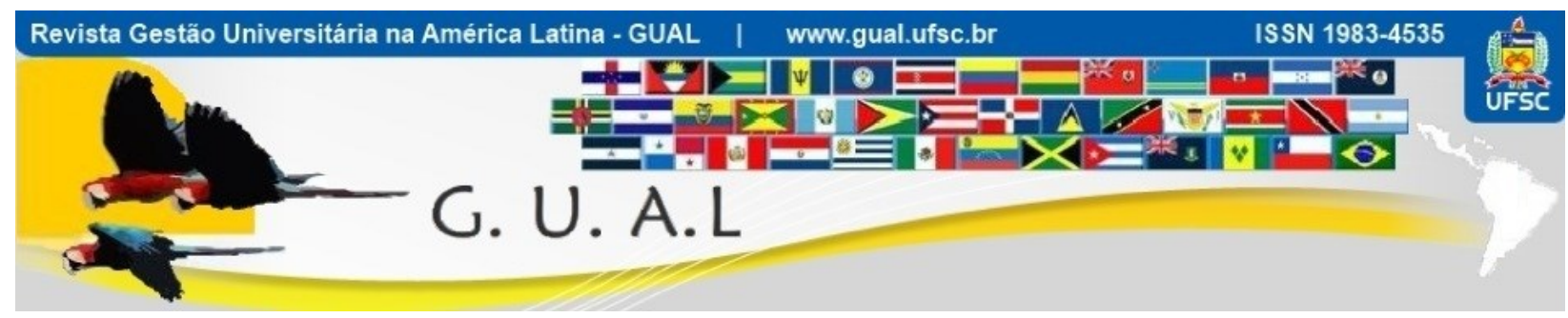

DOI: http://dx.doi.org/10.5007/1983-4535.2016v9n2p192

\title{
PARADOXOS NA IDENTIFICAÇÃO ORGANIZACIONAL COM UMA INSTITUIÇÃO DE ENSINO SUPERIOR
}

\section{PARADOXES IN ORGANIZATIONAL IDENTIFICATION WITH AN HIGHER EDUCATION INSTITUTION}

Edson Roberto Scharf, Doutor Universidade Regional de Blumenau - FURB artigoes@gmail.com

Recebido em 10/novembro/2014

Aprovado em 25/fevereiro/2016

Sistema de Avaliação: Double Blind Review

Esta obra está sob uma Licença Creative Commons Atribuição-Uso. 


\title{
RESUMO
}

O objetivo deste estudo foi confirmar a identificação de alunos de um curso de Mestrado em Administração com a marca da universidade em que estudam, com base no trabalho de Celsi e Gilly (2010). É de natureza descritiva com caráter indutivo. Os resultados apontaram para o entendimento de que a comunicação eleva a visibilidade da instituição; para a compreensão de que o sucesso futuro do estudante depende do sucesso da organização; para a sensação de bem-estar do aluno quanto aos valores e crenças veiculados na comunicação; e para a disposição dos estudantes em empreender esforços além dos normais para valorizar a organização. Apesar disto, a comunicação institucional gera um baixo índice de bem-estar e orgulho aos estudantes, quando perguntados se comentariam sobre ela com pessoas próximas. Neste estudo, tal efeito paradoxal foi denominado de 'espelho negro', com os estudantes absorvendo informação importante da comunicação, entendendo e concordando com o seu conteúdo, mas não o divulgando.

Palavras-chave: Marketing em instituições de ensino superior. Identificação organizacional. Comunicação. Branding. Pós-graduação.

\begin{abstract}
The aim of this study was to know the organizational identification of master students at an higher education institution in Brazil, in support of educational management. Based on the study by Celsi and Gilli (2010), with an exploratory inductive character, participated in the survey all students in the course. The results point to the understanding that the communication raises the visibility of the institution, to the realization that the future success of students depends on the success of the organization, for a sense of well-being of the students about the values and beliefs conveyed in communication; and that students are willing to make efforts beyond the normal value for the organization. Nevertheless, this same communication generates a low level of wellbeing and pride for students, when asked if they would comment on it with those close. In this study, the effect was called 'Black Mirror', with students absorbing important information communication, understanding and agreeing with its contents, but not to sharing. The results obtained allow the manager of the institution to plan more adequately their communication with the community of potential students.
\end{abstract}

Keywords: Higher education institution. Marketing management at HEI. Organizational identity. Branding. Advertising. Master of business administration. 


\section{INTRODUÇÃO}

Consumidores de bens e serviços buscam referências e informações para avaliar adequadamente as alternativas que possam atender as suas necessidades. Dentre outras análises, a marca do produto é fundamental neste processo. Ela aumenta a preferência da intenção de compra (PAPPU et al. 2006), sendo a mídia uma ferramenta eficiente para a sua comunicação.

Comum em organizações comerciais, também as instituições de ensino superior utilizam os esforços de gestão da marca, o denominado branding, para obter vantagens competitivas. Há, inclusive, discussão sobre a ideia de que a imagem e a reputação das universidades podem ser fatores mais importantes do que a qualidade do ensino em si (MAZZAROL, 1998). Diversos autores (BERRY, 2000; KLAUS e MAKLAN, 2007) apoiam o branding como importante para as organizações de serviço devido ao fato de que os ativos intangíveis dirigem cada vez mais as transações comerciais. A divergência em relação a essas afirmativas ocorre nos estudos de Bunzel (2007), ao sugerir que os esforços de branding de algumas universidades podem não ter muita clareza.

Quando se candidata a um curso stricto sensu, o estudante avalia aspectos como a aderência à carreira profissional ou o sentimento de segurança que a instituição confere, dentre outros. A marca de uma instituição de ensino superior facilita a escolha dos candidatos. Os esforços mercadológicos para comunicar os valores, o orgulho de pertencimento e as promessas da universidade têm papel fundamental na construção da identificação organizacional. Diversos autores (BULOTAITE, 2003; BUNZEL, 2007) defendem que a criação de uma marca de sucesso deve abordar aspectos como um produto efetivo, uma identidade distinta das demais universidades, o valor agregado e sua a percepção de relevância. Valor pode ser comunicado por meio de distinção, capital humano, reputação, dentre outros componentes, e pode ser percebido por intermédio da demonstração de conquistas, trabalhos criativos, qualidade nos serviços ou outros enfoques de disseminação.

Uma gestão escolar de qualidade se baseia em um modelo que faz uso intensivo do capital humano (CHAPLEO, 2010). Para o autor, não é possível uma organização ser orientada ao mercado sem ser orientada às pessoas: quando há um adequado funcionamento interno, funcionários e professores se esforçarão para atender aos objetivos organizacionais. Neste sentido, diversos autores (BITITCI et al. 2004; CELSI e GILLY, 2010; SCHARF, 
2012) argumentam que há um sentimento positivo relacionado às organizações quando a comunicação mercadológica é relacionada ao capital humano.

O desenvolvimento da marca para instituições de ensino superior é inerentemente mais complexo e as técnicas convencionais de branding são inadequadas neste segmento, tendo sido trazidas das organizações comerciais, mas não necessariamente adaptadas (JEVONS, 2006). Para Hemsley-Brown e Goonawardana (2007), a despeito do fato de terem sido feitos grande número de estudos que examinam a imagem e a reputação, a noção de branding mal deixou a sua marca na literatura de marketing das instituições de ensino superior. Alguns autores (CHAPLEO, 2010; BUNZEL, 2007) entendem que há uma lacuna que certamente deve ser pesquisada, constituída de uma proposta de identidade de marca não muito clara e o estabelecimento daquilo que as universidades buscam alcançar com o esforço de branding.

O objetivo deste estudo foi confirmar a identificação de alunos de um curso de Mestrado em Administração com a marca da universidade em que estudam, com base no trabalho de Celsi e Gilly (2010). A principal contribuição desta pesquisa é o seu contexto. Enquanto existe quantidade considerável de trabalhos sobre o que constitui uma marca de sucesso em organizações comerciais, estudos que buscam relacionar a comunicação com a identificação da marca de uma instituição de ensino superior ainda são incipientes. Na medida em que os resultados foram emergindo da pesquisa de campo, esta lacuna contextual a respeito da identificação organizacional foi se confirmando segundo a revisão teórica, a seguir apresentada.

\section{A DISSEMINAÇÃO DA COMUNICAÇÃO PELOS PARES}

A comunicação sempre foi o destino final dos esforços de marketing de uma organização; agora, ela é o início da relação, pois o consumidor é reconhecido como potencializador da mensagem publicitária para outros públicos. Quando disseminada, a comunicação tem a importante função de criar vínculos com a marca que, por sua vez, ampliam a sensação geral de satisfação. Segundo Brown et al. (2005), estudos mostram que a satisfação do consumidor é significantemente associada à intenção de prover recomendações.

$\mathrm{Na}$ decisão de compra, as informações coletadas são, principalmente, de duas formas: as do esforço tradicional de marketing, como propaganda e merchandising, em que alguns pesquisadores (WANG et al. 2009; TSANG et al. 2004) defendem que os principais fatores que contribuem para que o consumidor perceba a comunicação são a credibilidade, a 
demografia, o entretenimento, a interatividade, a irritação e o materialismo, e as de teor sóciocultural. Estas são as fontes não comerciais de informação, obtidas com a experiência de uso do produto, comentários de membros da família ou de vizinhos e críticas em revistas (APEJOYE, 2013).

A literatura de marketing possui ampla documentação a respeito de como os tomadores de decisão são afetados pelos pares e que a similaridade entre os emissores e receptores pode incrementar a persuasão da comunicação (BROWN et al. 2005; WANG et al. 2009). Conhecido como word of mouth (WoM) na literatura de marketing, para Brown et al. (2005) provavelmente é o mais antigo dos mecanismos pelo qual as opiniões sobre marcas são desenvolvidas e expressas. Alguns veículos são mais propícios a esse compartilhamento, pois todo consumidor pode distribuí-las instantaneamente entre dezenas de amigos pela internet (NORIEGA e BLAIR, 2008).

Em estudo sobre as influências da comunicação midiática, Zanchetta Jr. (2010) desenvolveu uma gradação da complexidade de entendimento dos estudantes em relação aos contextos que os cercam, com observação daquilo que os preocupa cotidianamente. Com destaque pontual dos circuitos de informação do aluno, é possível, segundo o autor, ver as influências e as relações estabelecidas por ele quando da seleção e da apropriação das informações noticiosas, num determinado momento por meio de três planos: interpessoal, organizacional e sistêmico. O plano interpessoal auxilia de forma decisiva a compor as agendas temáticas individuais. Neste sentido, parentes, amigos, colegas de escola, com influência da mídia, da igreja e de outros grupos são decisivos para seleção, entendimento e uso da informação no cotidiano. Consumidores citaram que 'pessoas' são fontes mais confiáveis de informações e troca de ideias do que a propaganda (WANG et al. 2009; TAMBORINI et al. 2010; APEJOYE, 2013).

O compartilhamento daquilo que vê ocorre quando o consumidor acha o conteúdo da mensagem envolvente, interessante e, na maioria das vezes, que se identifique com seus próprios valores (TSANG et al. 2004; NORIEGA e BLAIR, 2008). Portanto, a divulgação como um ato costumeiro não acontece caso não ocorra esta identificação. Para Osmonbekov et al. (2009) existe uma correlação positiva entre o nível de expertise que o consumidor tem e o nível de pensamento crítico. O conhecimento do produto, por meio da experiência, permite ao consumidor pensar mais criticamente sobre ele quando apresentado ao esforço publicitário. Pesquisas indicam, também, que sentimentos negativos são causados pela percepção dos 
consumidores a respeito de manipulação e persuasão subliminar feitas pelos publicitários (OSMONBEKOV et al. 2009).

\section{A IDENTIFICAÇÃO ORGANIZACIONAL E A MARCA NAS INSTITUIÇÕES DE ENSINO}

A identificação com uma organização, segundo Elsbach e Kramer (1996) tem duas dimensões: a interna (a que é percebida por seus membros, decorrente da autopercepção ou da autoimagem) e a externa (decorrente da maneira pela qual a organização é percebida por aqueles que com ela se relacionam). Para diversos autores (ANANA e NIQUE, 2010; CELSO e GILLY, 2010; APEJOYE, 2013; PUUSA et al. 2013) existem elementos que auxiliam a identificação externa, como a propaganda para a marca ou a distinção a ela conferida, que pode criar valor no entendimento do público.

Dada a concorrência competitiva nos diversos segmentos, a marca pode ser o elemento que diferencia uma organização da outra (KLAUS e MAKLAN, 2007; ANANA e NIQUE, 2010). Uma marca tem importante valor no planejamento de marketing (RUST et al. 2004) e aumenta a preferência da intenção de compra (PAPPU et al. 2006). No contexto dos serviços a marca é um ativo cuja importância cresce em relação aos bens (BERRY, 2000; KLAUS e MAKLAN, 2007), pois as avaliações são subjetivas, antes e depois do consumo.

Alunos também são expostos aos esforços mercadológicos das marcas, incluindo as das IES em que estudam, e podem estar prontos a considerar seu papel como stakeholders, refletindo uma imagem organizacional forte a partir da sua própria identidade (SCOTT e LANE, 2000; KIMPAKORN e TOCQUER, 2009; PUUSA et al. 2013). A menção do nome de uma universidade imediatamente evoca associações, emoções e imagens, e o papel da marca da universidade é construir, gerir e desenvolver essas impressões (BULOTAITE, 2003).

Há uma série de fatores que têm impulsionado a marca do ensino superior, como a diferenciação competitiva, o alcance do status de universidade e a incompatibilidade entre as promessas da marca e a entrega dos benefícios (BUNZEL, 2007; CHAPLEO, 2010). Sendo a instituição de ensino uma organização basicamente prestadora de serviços, a identidade da marca é o fator diferencial em situações competitivas (WHEELER et al. 2006). As universidades também podem ser medidas pelo conceito do apelo da personalidade da marca (FRELING et al. 2011). É composto por três dimensões: favorabilidade (a medida em que os consumidores levam em conta, de forma positiva, a personalidade da marca); originalidade (a 
medida em que os consumidores percebem a personalidade da marca como distinta de outras marcas na mesma categoria); e clareza (a medida em que a personalidade de uma marca é reconhecível para os consumidores). Neste sentido, as instituições de ensino superior precisam ser gerenciadas como marcas corporativas (WHELAN e WOHLFEIL, 2006), embora a implementação de técnicas de branding em alguns setores, incluindo o de ensino, ainda pode ser objeto de resistência (CHAPLEO, 2010). Para organizações complexas, como universidades, Bulotaite (2003) advoga que o branding simplifica esta complexidade, promovendo atração e lealdade para a organização.

As organizações de serviço frequentemente seguem estratégias monolíticas de branding, com os consumidores sendo levados a ter expectativas uniformes dos serviços ofertados (CHAPLEO, 2007), embora uma das funções primárias do branding seja monitorar e ajustar as interações entre a organização e seus stakeholders (HEMSLEY-BROWN e GOONAWARDANA, 2007; SCHARF, 2012). Considerando que a escola também é uma empresa, Paro (2010) sugere que a administração, ao cuidar da utilização racional dos recursos, seja feita por múltiplas pessoas.

\section{MÉTODO}

Este estudo é de natureza descritiva de caráter indutivo. Com o uso de questionários e escala do tipo Likert, teve como objetivo confirmar a comunicação como ação estratégica para a identificação com a marca da universidade em que alunos de um curso de Mestrado em Administração estudam.

\subsection{AMOSTRA}

Foi composta da totalidade dos alunos no curso de pós-graduação stricto sensu, Mestrado em Administração, no corrente ano (55 alunos), de uma das mais importantes universidades do estado de Santa Catarina, no sul do Brasil. Os participantes responderam anonimamente o questionário, tomando aproximadamente 20 minutos do seu tempo.

\subsection{PROCEDIMENTO}

Os alunos obtiveram o diploma de graduação em variadas instituições de ensino, sendo que oito deles na instituição-objeto deste estudo. Todos conheciam a organização antes de decidir cursar o mestrado, por meio da comunicação, como propaganda, folders, outdoors, 
palestras explicativas e artigos em jornais. O questionário foi respondido durante a disciplina de Estratégias de Marketing. As questões formuladas foram adaptadas do trabalho Measurement Items and Factor Loadings (CELSI e GILLY, 2010), que contém os seguintes construtos: a. Precisão da promessa da propaganda; b.Identificação organizacional; c.Congruência de valores; d.Efetividade da propaganda; e.Orgulho; f. Foco no consumidor; g.Precisão da representação dos funcionários; h.Precisão da representação dos alunos. Neste estudo, foram pesquisados os cinco primeiros construtos. Esta escolha ocorre por se encontrarem conectados diretamente ao tema do trabalho: a comunicação. $O$ construto 'identificação organizacional' é de entendimento de Anana e Nique (2010); Celsi e Gilly (2010); Freling et al. (2011); e de Peterson e Jeon (2010), como sendo diretamente conectado aos esforços de comunicação. Os construtos 'congruência de valores' e 'orgulho' são de entendimento de Bunzel (2007); e de Gilly e Wolfinbarger (1998) como relacionados ao mesmo aspecto. Os construtos 'promessa da propaganda' e 'efetividade da propaganda' são elementos relacionados ao tema, conforme diversos autores (GILLY e WOLFINBARGER, 1998; AILAWADI et al. 2003; PETERSON e JEON, 2010; FRELING et al. 2011; APEJOYE, 2013). Os demais construtos são complementares ao objetivo de estudo, sendo desconsiderados neste trabalho.

\subsection{OS CONSTRUTOS AVALIADOS}

Este estudo foi desenvolvido com base em cinco construtos-chave, adaptados de Celsi e Gilly (2010):

a) Precisão da Promessa da Propaganda: a propaganda produz significado para o público externo, formado pelos consumidores, e para o público interno, formado pelos membros da organização (CELSI e GILLY, 2010). Em qualquer dos dois casos, o aluno pode ser considerado uma figura central, pois ao comprar o serviço de ensino atua como consumidor e ao usufruir daquilo que comprou, é um membro que atua dentro dela. Como esforço mercadológico, a propaganda é, provavelmente, o mais visível e memorável elemento da comunicação organizacional externa, e também o mais controlável (EWING et al. 2002; APEJOYE, 2013).

b) Identificação Organizacional: os esforços de pesquisa para entender os efeitos da comunicação na audiência interna têm sido limitados, segundo Celsi e Gilly (2010). A comunicação de determinado curso pode ser dirigido a um público selecionado, mas tem 
repercussões significativas nos outros públicos, segundo os autores. As mensagens produzem significado tanto para o grupo para o qual a comunicação foi criada quanto para os outros públicos, criando uma identificação com a instituição de ensino. Quando as crenças e as atitudes das pessoas são próximas às da instituição, ocorre a identificação organizacional.

c) Congruência de Valores: a congruência de valores ocorre quando há similaridade entre os valores pessoais e os comunicados. Alguns dos valores comumente citados na comunicação são a criatividade, a alegria, o trabalho em grupo ou a qualidade dos serviços (CELSI e GILLY, 2010). A congruência de valores se conecta a resultados que incluem a satisfação, o comprometimento, o engajamento emocional e a confiança, elementos estes que podem apoiar positivamente a visão da organização, por meio da comunicação (VERPLANKEN e HOLLAND, 2002).

d) Efetividade da Propaganda: conjuntamente com a promessa da propaganda e a congruência de valores, este construto mostra se os estudantes acreditam que a comunicação é bem-sucedida em obter a atenção do público-alvo e conseguir gerar vendas. Para Celsi e Gilly (2010) os funcionários são especialmente suscetíveis na avaliação dos anúncios de suas próprias organizações, uma vez que eles têm uma participação na consecução dos objetivos organizacionais.

e) Orgulho: o orgulho pode encorajar comportamentos onde as pessoas se tratem com dignidade e respeito (MICHIE, 2009). Os funcionários podem se motivar a dar suporte aos processos de foco no cliente quando se sentem orgulhosos da comunicação da organização em que trabalham (CHUN e DAVIES, 2006).

\subsection{VALIDAÇÃO DOS DADOS}

Os dados obtiveram índices de confiabilidade adequados ao estudo. $\mathrm{O}$ alfa de Cronbach geral encontrado foi de 0,969 . O menor valor foi de 0,966 para o construto Orgulho. Portanto, todos os construtos demonstraram índices adequados de confiabilidade, já que o valor mínimo para um construto ser considerado confiável é de 0,70 (BOLLEN, 1989). A média não é representativa, pois a dispersão é relativamente alta, conforme tabela 1. 
Tabela 1 Estatística descritiva (para o mínino de 1 e máximo de 7 na escala Likert).

\begin{tabular}{|c|c|c|c|c|}
\hline Construtos-chave & $\mathbf{N}$ & Média & Desvio-padrão & Variância \\
\hline Identificação organizacional & 220 & 5,89 & 1.499 & 2.247 \\
\hline Precisão da promessa da propaganda & 165 & 4,87 & 1.516 & 2.299 \\
\hline Congruência de valores & 165 & 4,94 & 1.652 & 2.728 \\
\hline Efetividade da propaganda & 220 & 5,48 & 1.491 & 2.223 \\
\hline Orgulho & 165 & 4,48 & 1.847 & 3.410 \\
\hline
\end{tabular}

Para minimizar este efeito, se calculou a média descritiva representada pelos números das variáveis de cada dimensão estudada, se utilizando os graus de 'concordância', 'concordância forte' e 'concordância total' da escala de Likert. Os números encontrados se referem à média positiva da escala, o que permite visualizar os aspectos adequadamente avaliados pelos alunos. A correlação de Pearson indicou que o construto Efetividade da Propaganda é significativo em relação ao de Identificação Organizacional, conforme demonstrado na tabela 2.

Tabela 2 Correlação de Pearson.

\begin{tabular}{|c|c|c|c|}
\hline Construtos-chave & $\begin{array}{c}\text { Correlação de } \\
\text { Pearson } \\
\text { (Identificação } \\
\text { organizacional) }\end{array}$ & $\begin{array}{c}\text { SIG (2-tailed) } \\
\text { Sig. }<\mathbf{0 , 0 5}\end{array}$ & Coeficiente \\
\hline Precisão da promessa da propaganda &, 065 &, 678 & Não significativo \\
\hline Congruência de valores &, 050 &, 246 & Não significativo \\
\hline Efetividade da propaganda &, 558 &, 000 & Significativo \\
\hline Orgulho &, 037 &, 237 & Não significativo \\
\hline
\end{tabular}

O teste $t$ obteve significância, conforme pode ser observado na tabela 3. As médias dos construtos avaliados são válidas.

Tabela 3 Teste $t$.

\begin{tabular}{|c|c|c|c|}
\hline $\mathbf{9 5 \%}$ - intervalo de confiança & $\boldsymbol{T}$ & df & Sig. (2-tailed) \\
\hline Identificação organizacional & 18.664 & 219 &, 000 \\
\hline Precisão da promessa da propaganda & 7.342 & 164 &, 000 \\
\hline Congruência de valores & 7.306 & 164 &, 000 \\
\hline Efetividade da propaganda & 14.740 & 219 &, 000 \\
\hline Orgulho & 3.331 & 164 &, 001 \\
\hline
\end{tabular}

\subsection{LIMITAÇÃO DA PESQUISA}

A literatura aponta para a falta de estudos desafiadores sobre o tema (CELSI e GILLY, 2010; CHAPLEO, 2010). Logo, é difícil medir o sucesso das instituições de ensino superior quanto à identificação com a sua marca quando há pouca literatura empírica de branding em relação a universidades. 
A amostra é outro limitador. Porém, deve ser observada com especial atenção a qualificação da mesma: o estudo foi realizado com a totalidade dos alunos de Mestrado em Administração em determinada instituição de ensino superior no ano. O perfil dos estudantes pode auxiliar a minimizar possíveis desvios. No Brasil, os candidatos a um curso de pósgraduação stricto sensu em Administração e Ciências Contábeis devem se submeter a uma prova de conhecimentos específicos (inglês, português, raciocínio analítico, raciocínio lógico e raciocínio quantitativo), aplicada por órgão nacional. Após a primeira fase, eliminatória, ocorrem duas outras, eliminatórias e classificatórias, administradas pelas próprias universidades: a análise dos currículos profissional e acadêmico, e uma entrevista com, no mínimo, dois professores do programa de pós-graduação. Estas atividades permitem avaliar o nível do seu envolvimento com disciplinas de Administração, as expectativas quanto ao curso, o comportamento dele em relação às atividades de pesquisa, e o seu conhecimento acadêmico de Administração. Estas atividades avaliativas, ainda que de caráter subjetivo, permitem reconhecer a amostra como sendo de opinião representativa.

\section{RESULTADOS}

Tipicamente, as pesquisas que relacionam consumidores e organizações tratam dos aspectos de percepção da imagem (AILAWADI et al. 2003; ANANA e NIQUE, 2010) ou da qualidade dos serviços (BERRY, 2000). Neste sentido, a literatura de branding pode ser dividida em termos de 'racional' e 'emocional', ou da perspectiva ampla 'racional mais emocional' (CHAPLEO, 2010). Estudos que têm como escopo relacionar o esforço de comunicação à identificação da marca de uma instituição de ensino superior ainda são incipientes. A seguir, as considerações acerca dos construtos mais relevantes para os alunos de um curso de Mestrado na identificação da marca de uma universidade.

\subsection{PRECISÃO DA PROMESSA DA PROPAGANDA}

Neste construto (ver tabela 4) foi perguntado se 'o produto ofertado era tão bom quanto o apresentado na propaganda', se obtendo uma média positiva de $74,55 \%$ (alfa de cronbach de 0,968$)$, e se 'as propagandas prometem exatamente o que a instituição entrega', com média positiva de $65,45 \%$ (alfa de cronbach de 0,967 ). As respostas apontam que a maior parte das pessoas relaciona a sua experiência pessoal com aspectos vistos na comunicação, confirmando estudos de Goldman e Papson (1994). 
Tabela 4 Dados agrupados do construto 'Precisão da promessa da propaganda'.

\begin{tabular}{|lccc|}
\hline & & & \\
& & & \\
\hline & Q_2__1 & Q_2_2 & Q_2_3 \\
\hline Média & 4,93 & 4,95 & 4,73 \\
\hline Desvio Padrão & 1,54 & 1,41 & 1,62 \\
\hline Coeficiente de variação (\%) & 31,21 & 28,44 & 34,17 \\
\hline
\end{tabular}

O entendimento dos estudantes de que a promessa é cumprida se relaciona com a proposta de valor da instituição e demonstra que a comunicação tem obtido resultados positivos quanto ao reconhecimento das competências comunicadas ao mercado. Diversos autores (WHELAN e WOHLFEIL, 2006; SCOTT e LANE, 2000; SRIVASTAVA et al. 1999) afirmam que a comunicação pode acelerar o processo de difusão da marca. Os funcionários - neste caso, os estudantes - que já estão fortemente identificados com a organização tendem a avaliar os anúncios dela de forma positiva (VERPLANKEN e HOLLAND, 2002; CHUN e DAVIES, 2006; CELSI e GILLY, 2010; HE e BROWN, 2013). Segundo Whelan e Wohlfeil (2006) instituições de ensino superior têm um diferencial competitivo quando são gerenciadas como marcas corporativas.

Os resultados também sugerem que as proposições e promessas feitas aos candidatos e estudantes dos cursos de Mestrado têm sido prontamente compreendidas, o que facilita a identificação com a organização. Para diversos autores (BULOTAITE, 2003; JEVONS, 2006; HEMSLEY-BROWN e GOONAWARDANA, 2007) a comunicação das instituições educacionais requer uma proposta única de venda, para facilitar a escolha dos estudantes em relação à universidade que pretendem estudar. Com este entendimento, o construto Precisão da Promessa da Propaganda auxilia o desenvolvimento de uma marca consolidada para a universidade estudada.

\subsection{IDENTIFICAÇÃO ORGANIZACIONAL}

A frase que afirmou que 'o aluno tem orgulho de dizer aos outros que estuda na instituição', obteve um percentual de $85,45 \%$ das respostas consideradas com média positiva (alfa de cronbach de 0,967$)$. O esforço das universidades em relação à marca tem o potencial para criar sentimentos fortes tanto quanto as marcas de outros segmentos (HEMSLEYBROWN e GOONAWARDANA, 2007; DOREN e CORRIGAN, 2008; HE e BROWN, 
2013). A chave para fazer isto com sucesso é criar uma identidade única de comunicação (BULOTAITE, 2003). Ao comunicar aos outros a marca da universidade em que estuda, com um sentimento de distinção, o aluno demonstra a identificação com a organização. Afinal, a experiência com uma marca é multidimensional (DILLARD et al. 2007; ANANA e NIQUE, 2010; FRELING et al. 2011). Os estímulos em relação a ela são causados pelo meio com o qual o consumidor obtém uma experiência pessoal (ANANA e NIQUE, 2010; CHAPLEO, 2010). Ver tabela 5.

Tabela 5 Dados agrupados do construto 'Identificação organizacional'.

\begin{tabular}{|lcccc|}
\hline & \multicolumn{3}{c|}{ Identificação organizacional } \\
\cline { 1 - 3 } & Q_5_1 & Q_5_2 & Q_5_3 & Q_5_4 \\
\hline Média & 5,93 & 6,07 & 6,04 & 5,51 \\
\hline Desvio Padrão & 1,39 & 1,41 & 1,56 & 1,60 \\
\hline Coeficiente de variação (\%) & 23,38 & 23,26 & 25,89 & 28,99 \\
\hline
\end{tabular}

É de se observar, porém, estudos de Dillard et al. (2007), que indicam que investimentos em propaganda apresentam retornos decrescentes em indicadores reais de efetividade, embora não apresentem explicações de teor comportamental.

A afirmativa 'o aluno se importa com o destino da universidade', obteve percentual superior $(89,09 \%)$ nas respostas consideradas positivas (alfa de cronbach de 0,967). Consumidores satisfeitos com o serviço prestado podem alavancar os efeitos da marca ao defenderem o uso de uma específica (KIMPAKORN e TOCQUER, 2009). Neste caso, a relação do estudante com a instituição de ensino superior não é apenas do tipo fornecedorcliente, demonstrando a sua lealdade ao afirmar que se importa com o destino da organização.

As questões seguintes deste construto, cujas afirmativas foram 'o sucesso desta universidade é o meu sucesso' $(85,45 \%$ e alfa de cronbach de 0,969$)$ e 'estou disposto a fazer um esforço além do normal para valorizar a instituição' (80,00\% e alfa de cronbach de 0,968), são exemplares ao demonstrar que a crença em uma organização pode auxiliar o reconhecimento da sua marca por meio do capital humano nela envolvido (BITITCI et al. 2004; SCHARF, 2012). Em um processo de branding é necessário o elemento comportamental identificado com a organização, pois segundo diversos autores (BULOTAITE, 2003; CHAPLEO, 2007; CELSI e GILLY, 2010; CHRISTENSEN e 
CORNELISSEN, 2011) funcionários e alunos de uma universidade mostram como percebem a marca por meio das suas atitudes em relação à instituição.

A identificação com a organização é uma das bases para a escolha de um envolvimento tão longo quanto o que ocorre com uma instituição de ensino. $\mathrm{O}$ fato de sentirse bem em dizer que frequenta determinada instituição, de estar disposto a esforços maiores para valorização da organização ou de acreditar que o sucesso da universidade é, também, o seu próprio sucesso, demonstra a sua identificação com ela.

O construto da identificação organizacional foi o que obteve as médias positivas mais altas na escala adotada.

\subsection{CONGRUÊNCIA DE VALORES}

Este construto (ver tabela 6) traz as afirmativas 'eu tenho orgulho dos valores expressos na propaganda da instituição' (70,91\% e alfa de cronbach de 0,966); 'os valores organizacionais insinuados pela propaganda são os valores e crenças que eu daria destaque na propaganda' (63,64\% e alfa de cronbach de 0,966); e 'os valores apresentados na propaganda são similares aos meus valores' $(65,45 \%$ com alfa de cronbach de 0,967$)$. Comumente, as pessoas se mantêm em determinada organização por entender que os valores que aspiram e praticam são os mesmos adotados pela empresa em que trabalham. No caso de uma instituição de ensino, não há a relação de emprego, porém, a natureza é semelhante: o estudante se mantém durante um longo período na mesma, está sujeito às regras da organização e deve contribuir com ela. Neste sentido, Wheeler et al. (2006) sugere que isto concentra todos os esforços na direção dos objetivos organizacionais e pessoais, que devem estar alinhados para satisfação mútua, podendo gerar lealdade. Neste sentido, alguns dos influenciadores da lealdade dos estudantes em relação às IES se referem ao compromisso dos alunos com os seus próprios objetivos (HENNIG-THURAU et al. 2001); à reputação da instituição (HELGESEN e NESSET, 2007; CHRISTENSEN e CORNELISSEN, 2011); e à imagem institucional (HEMSLEY-BROWN e GOONAWARDANA, 2007; ANANA e NIQUE, 2010). 
Tabela 6 Dados agrupados do construto 'Congruência de valores'.

\begin{tabular}{|lccc|}
\hline \multicolumn{3}{|c|}{ Congruência de valores } \\
\hline & Q_6_1 & Q_6_2 & Q_6_3 \\
\hline Média & 5,02 & 4,80 & 5,00 \\
\hline Desvio Padrão & 1,81 & 1,66 & 1,49 \\
\hline Coeficiente de variação (\%) & 36,08 & 34,58 & 29,81 \\
\hline
\end{tabular}

No entanto, é de se observar uma distância entre os valores obtidos no construto 'Identificação Organizacional' com os do construto 'Congruência de Valores'. Para a obtenção de uma marca consolidada, os valores das pessoas envolvidas na sua construção devem estar muito próximos dos valores comunicados e, neste estudo, se demonstra uma lacuna. Marca de sucesso é um construto multidimensional baseado nos critérios de negócio e consumo. Esta visão, segundo Anana e Nique (2010), sugere que a característica que define a diferença entre uma marca de sucesso e uma que fracassou é que a marca de sucesso mostra um alto nível de congruência entre os valores desenvolvidos pela organização para as suas marcas e as necessidades racionais e emocionais dos consumidores.

\subsection{EFETIVIDADE DA PROPAGANDA}

Foram tratados aspectos relacionados aos resultados obtidos pela propaganda, e a afirmativa que obteve a média positiva isolada mais alta foi a que diz que 'a propaganda efetivamente eleva a visibilidade da universidade' (média positiva de $85,45 \%$, com alfa de cronbach de 0,968). Este aspecto, em conjunto com os aspectos dos construtos de 'Identificação Organizacional' e de 'Congruência de Valores', corrobora a ideia central de Celsi and Gilly (2010) de que muitos alunos se identificam com determinada instituição de ensino em função do contato que tiveram com a comunicação da marca. Ver tabela 7.

Tabela 7 Dados agrupados do construto 'Precisão da propaganda'.

\begin{tabular}{|lcccc|}
\hline & \multicolumn{3}{c|}{ Efetividade da propaganda } \\
\cline { 1 - 3 } & Q_8_1 & Q_8_2 & Q_8_3 & Q_8_4 \\
\hline Média & 5,62 & 5,42 & 5,91 & 4,98 \\
\hline Desvio Padrão & 1,55 & 1,46 & 1,47 & 1,37 \\
\hline Coeficiente de variação (\%) & 27,51 & 26,98 & 24,86 & 27,45 \\
\hline
\end{tabular}


A alta média positiva demonstra que a universidade tem obtido resultados concretos com a comunicação, tanto em efetividade da venda quanto no aceite por parte dos estudantes. Embora normalmente os objetivos primários da propaganda sejam persuadir à venda, informar, lembrar e reforçar, nem sempre a imagem da marca tem o mesmo apelo para todos os consumidores (CHUN e DAVIES, 2006; SCHARF, 2012). Autores diversos acreditam que a imagem da marca é o reflexo dos resultados da comunicação, dentre outros esforços, e que esta imagem gerada contribui para atitudes favoráveis à marca (SRIVASTAVA et al. 1999; AILAWADI et al. 2003). Neste sentido, a média positiva deste construto é altamente favorável à identificação organizacional.

Porém, quando utilizada a Correlação de Pearson se verificou que o construto 'Efetividade da Propaganda' apresentou correlação significativa (tabela 2), podendo indicar uma ligação comum entre os eventos, segundo Bollen (1989), ainda que o método não deva ser utilizado para fazer predições. Assumindo que a correlação seja importante e que o construto de Efetividade da Propaganda não esteja em consonância com os demais, é possível desenvolver algumas suposições com base na literatura de marketing e, mais especificamente, na literatura de branding.

A primeira diz respeito ao que pode ser considerado como ação efetiva na comunicação. Para Peterson e Jeong (2010), a comunicação pode acelerar a velocidade da resposta de consumo e induzir a penetração no mercado mais rapidamente. As questões deste construto se relacionam ao aumento de vendas, aceitação da mensagem, elevação da visibilidade e efetividade dos resultados. O estudante, pela natureza da sua ligação com a universidade, não tem condições de avaliar objetivamente um destes aspectos (venda), embora possa ter uma noção ao se deparar com salas de aula repletas, novos edifícios sendo construídos e o movimento nos campi da instituição. No entanto, nos demais, há a possibilidade de análise porque são aspectos que dizem respeito à avaliação comum, entre os próprios estudantes como, por exemplo, quando a propaganda é comentada positivamente no seu grupo ou o nome da instituição é considerado como importante pelo seu grupo de interação.

A segunda suposição ocorre ao se observar os resultados obtidos no construto 'Orgulho', a seguir, que obteve a média positiva mais baixa. Os sentimentos de bem-estar ao comentar sobre a comunicação com outras pessoas e de orgulho da instituição são aspectos diretamente ligados à efetividade do esforço realizado. Para Tamborini et al. (2010) o 
sentimento de satisfação com a comunicação é uma atitude em direção à experiência do entretenimento, completada com as dimensões afetiva, comportamental e cognitiva.

E uma terceira pode ser discutida ao se observar o conteúdo da mensagem publicitária como fonte de efetividade dos resultados. Em uma avaliação criteriosa, observando o questionário adaptado de Celsi e Gilly (2010) utilizado nesta pesquisa, especificamente o aspecto relativo à efetividade dos resultados, os estudantes podem estar sinalizando que a comunicação da universidade é subjetiva, se apoiando em valores, experiências e expectativas futuras. É um raciocínio comum dos esforços publicitários no segmento, com a intenção de promover um reconhecimento positivo em relação ao que espera o estudante na conclusão do curso (sucesso, melhor colocação profissional, destaque na área de atuação). No entanto, a propaganda pode ser desenvolvida com mais informações para o auxílio à busca de determinados elementos para compra, não necessariamente para contribuir com a experiência do consumidor. Em estudo de Franke et al. (2004) se confirmou que o incremento de informações em anúncios que relatavam alguma experiência não aumentou o nível de leitura do anúncio e, em alguns casos, até o diminua. Somado a este fato, os estudos de Bunzel (2007) apontam para a existência de amplas evidências de que os consumidores evitam comerciais e outras formas de comunicação.

\subsection{ORGULHO}

Os valores de média positiva mais baixos foram expressos neste construto (ver tabela 8). A primeira afirmativa foi de que 'a propaganda me faz orgulhoso da instituição de ensino' $(65,45 \%$ com alfa de cronbach de 0,966$)$. A segunda comentou que 'vendo a propaganda sinto-me bem em relação à universidade' $(70,91 \%$ com alfa de cronbach de 0,966$)$ e a terceira afirmativa trouxe a frase 'eu gosto de contar aos outros sobre as propagandas da universidade' (36,36\% com alfa de cronbach de 0,968). De acordo com pesquisas de diversos autores (GILLY e WOLFINBARGER, 1998; HELGESEN e NESSET, 2007; MICHIE, 2009), os funcionários têm orgulho de propaganda que evidencia o sucesso organizacional, em que familiares e amigos a discutem. Também realçam o orgulho organizacional quando a propaganda é julgada de sucesso e o minimizam quando entendem que a propaganda não demonstra este sentimento. Embora o aluno não seja funcionário, no momento em que entra na universidade assume algumas posturas de envolvimento que são próximas àquelas adotadas pelos funcionários da organização (KIMPAKORN e TOCQUER, 2009). Trabalhos 
intermediários (artigos da disciplina, discussões em grupo ou desenvolvimento de atividades intelectuais) e trabalhos finais (artigos científicos, trabalho de dissertação ou o desenvolvimento de produção intelectual) são produtos que auxiliam a boa avaliação externa da instituição.

Tabela 8 Dados agrupados do construto 'Orgulho'.

\begin{tabular}{|lccc|}
\hline & & Orgulho \\
& & & \\
\hline & Q_1_1 & Q_1_2 & Q_1_3 \\
\hline Média & 4,80 & 5,04 & 3,60 \\
\hline Desvio Padrão & 1,74 & 1,70 & 1,80 \\
\hline Coeficiente de variação (\%) & 36,17 & 33,74 & 50,03 \\
\hline
\end{tabular}

A partir de uma ampla análise de estudos de comportamentos em relação a marcas, Puusa et al. (2013) sugerem que a marca traz um reforço simbólico nela, além da utilidade, aquela que ocorre pelo próprio uso do bem ou do serviço. Assim, o simbolismo emanado pela marca pode aumentar as taxas relacionadas ao comportamento frequente no meio social, dirigindo os consumidores a escolher determinada marca em função do seu simbolismo. No entanto, os valores das médias demonstram uma lacuna ocorrida na comunicação em relação à construção de uma identificação organizacional adequada. Mais especificamente, o fato de os estudantes não comentarem entre si e com os demais o conteúdo das propagandas da universidade em que estudam pode apontar um distanciamento entre o que prega a comunicação e o sentimento para o qual é remetido o estudante. É possível que o raciocínio criativo da propaganda não esteja motivando o estudante. Neste caso, mudanças na estratégia de redação publicitária são desejadas. Autores (WHELAN e WOHLFEIL, 2006; PETERSON e JEONG, 2010) sugerem que a criatividade na propaganda é altamente valorizada por sua habilidade em ganhar a atenção dos consumidores e valorizar as marcas.

Considerando os resultados positivos obtidos nos construtos de 'Identificação Organizacional', de 'Precisão da Promessa da Propaganda' e de 'Efetividade da Propaganda', parece bastante aceitável que o formato da propaganda esteja inadequado para mover os estudantes rumo a um sentimento de orgulho em relação à universidade em que estudam. Para Peterson e Jeong (2010), a criatividade da comunicação é tão ou mais importante do que os investimentos feitos nela, em algumas instâncias. 


\section{CONCLUSÕES}

A comunicação é uma ação mercadológica que pode conduzir os consumidores à identificação organizacional.

Neste estudo, os estudantes do curso de pós-graduação stricto sensu, Mestrado em Administração, de uma instituição de ensino superior no Brasil, estado de Santa Catarina, afirmam que a comunicação eleva a visibilidade da instituição e que compreendem que seu sucesso futuro é dependente do sucesso da organização. Em parte, isto se deve ao fato de se sentirem à vontade com os valores e as crenças veiculados nos esforços de comunicação, tais como propaganda, eventos e redes sociais, sendo estes similares aos seus. E também por estarem dispostos a empreender esforços além dos normais para auxiliar a valorizar a organização. Apesar disto, esta mesma comunicação gera um baixo índice de bem-estar e orgulho aos estudantes, quando perguntados se comentariam sobre ela com as pessoas próximas.

Embora paradoxal, é uma situação explicável. Ainda que se sinta identificado com a instituição de ensino, pode não se identificar totalmente com o que é comunicado, do ponto de vista estético ou de conteúdo. Como exemplo, uma pessoa pode comprar o automóvel Porsche Panamera Turbo em função dos aspectos de sofisticação (considerado um atributo emocional); no entanto, sua comunicação é evidentemente voltada aos atributos funcionais de desempenho do bem. Nesta situação, o comprador se identifica com os aspectos ínsitos da comunicação, não com os explícitos, embora concorde com estes. Portanto, a lacuna observada no construto 'Orgulho' deve ser levada em consideração como uma peça fundamental no esforço de construção de identificação organizacional com a instituição de ensino superior.

As altas médias positivas alcançadas pelas afirmativas de que 'o produto é tão bom quanto o apresentado na propaganda', 'os valores expressos na propaganda são similares aos meus' e 'a propaganda efetivamente eleva a visibilidade da instituição', demonstram o nível de compreensão dos estudantes em relação às mensagens divulgadas na mídia. Ao mesmo tempo, a média positiva é muito baixa quanto à afirmação de 'gostar de contar aos outros sobre a propaganda da instituição', contribuindo para o entendimento de que não necessariamente as pessoas se sentem à vontade para assumirem o papel de porta-vozes da instituição, mesmo que concordem com a comunicação. 
Consideradas as limitações da pesquisa, este estudo permite afirmar que embora os estudantes de Mestrado compreendam a mensagem comunicada e se identifiquem com a instituição de ensino superior por meio dela, não comentam com outras pessoas sobre a comunicação: absorvem informação importante, entendem seu conteúdo, concordam com ele, mas não a divulgam. Neste estudo, tal efeito é denominado de 'espelho negro', uma alusão ao fato de os raios do sol serem absorvidos por áreas escuras, e não refletirem os mesmos. Para explicação do efeito, os raios do sol são os esforços de comunicação, a área escura é a mente do consumidor, e a ação de não refletir é a indisposição dos estudantes em repassar aquilo que viram na comunicação, ainda que um espelho devesse sempre ser refletivo. Por isto o uso do termo 'negro'.

O entendimento do processo de atitude do consumidor em relação à comunicação é fundamental para que os resultados desse tipo de ação sejam mensuráveis. O efeito 'espelho negro’ não necessariamente é danoso, pois confirma que parte do esforço publicitário surtiu resultado, basicamente pela compreensão do conteúdo da comunicação e da identificação organizacional. $\mathrm{O}$ fato dos estudantes absorverem a maioria das informações indicadas pela comunicação é positivo. Segundo os conceitos da Sociologia, os estudantes respondentes desta pesquisa estão, em sua maioria, concentrados no perfil da geração $\mathrm{Y}$, posto que nasceram entre 1978 e 1994. Como características, absorvem quantidade considerável de informações, são multitarefa e não se ocupam da lealdade a determinados símbolos nem hábitos como, por exemplo, o de ser divulgador daquilo que adotam no cotidiano. Como nasceram sem a necessidade de aprender a dominar a tecnologia, pois ela já estava presente em sua vida, formaram atitudes que alteraram completamente os hábitos de comunicação. Isto pode explicar a conveniência em não divulgar os fatos obtidos pela propaganda: a informação pode ser importante, mas como é compartilhada por todos, em tempo real, não há a necessidade de mais divulgação.

Este estudo comprova que a identificação organizacional, obtida por meio dos esforços de comunicação, é um aspecto de relevância para a marca de uma instituição de ensino superior, embora nem sempre esta mesma comunicação seja amplificada dos estudantes para outros grupos.

A contribuição gerencial é permitir aos gestores saber que a identificação e o entendimento do efeito 'espelho negro' são capitais para a administração de instituições de ensino superior, dada a competitividade vigente. Especificamente, porque permite 
investimentos de comunicação dirigidos aos grupos de formadores de opinião, minimizando a possibilidade de erros na trajetória de comunicação à marca.

Como contribuição acadêmica, o estudo permite ampla discussão acadêmica sobre os conceitos adotados em estudos relacionados à propaganda, especificamente a possibilidade de controle dos seus resultados, a construção de estratégias criativas dirigidas a determinados grupos de consumidores e o reconhecimento da marca obtido ou desejado por meio de ações estratégicas de comunicação. Ademais, são esperados estudos seguintes sobre ações complementares à propaganda para a obtenção da identificação organizacional, como o word of mouth, a publicidade, as redes sociais, os catálogos institucionais e os eventos.

\section{REFERÊNCIAS}

AILAWADI, K. L.; LEHMANN, D. R.; NESLIN, S. A. Revenue premium as an outcome measure of brand equity. Journal of Marketing, v.67, n.2, p. 1-17, 2003.

ANANA, E.; NIQUE, W. Perception-based analysis: An innovative approach for brand positioning assessment. Database Marketing and Customer Strategy Management, v.17, n.1, p. 6-18, 2010.

APEJOYE, A. Influence of celebrity endorsement of advertisement on students' purchase intention. Mass Communication and Journalism, v. 3, n. 3, 2013.

BERRY, L. L. Cultivating service brand equity. Journal of the Academy of Marketing Science, v.28, n.1, p. 128-139, 2000.

BITITCI, U. S.; MARTINEZ, V.; ALBORES, P.; PARUNG, J. Creating and managing value in collaborative networks. International Journal of Physical Distribution \& Logistics Management, v.34, n.3/4, p. 251-268, 2004.

BOLLEN, K. A. Structural equations with latent variables. New York: Wiley, 1989.

BROWN, T. J.; BARRY, T. E.; DACIN, P. A.; GUNST, R. F. Spreading the word: investigating antecedents of consumers' positive word-of-mouth intentions and behaviors in a retailing context. Journal of the Academy of Marketing Science, v.33, n.2, p. 123-138, 2005.

BULOTAITE, N. University heritage: an institutional tool for branding and marketing. Higher Education in Europe, v.28, n.4, p. 449-454, 2003.

BUNZEL, D. Universities sell their brands. Journal of Product \& Brand Management, v.16, n.2, 152-153, 2007. 
CELSI, M. W.; GILLY, M. C. Employees as internal audience: how advertising affects employees' customer focus. Journal of the Academy of Marketing Science, v.38, n.3, p. 520-529, 2010.

CHAPLEO, C. What defines "successful" university brands? International Journal of Public Sector Management, v.23, n.2, p. 169-183, 2010.

CHAPLEO, C. Barriers to brand building in UK universities? International Journal of Nonprofit and Voluntary Sector Marketing, v.12, p. 23-32, 2007.

CHRISTENSEN, L. T.; CORNELISSEN, J. Bridging corporate and organizational communication: Review development and a look to the future. Management Communication Quarterly, v. 25, n. 3, p. 383-414, 2011.

CHUN, R.; DAVIES, G. The influence of corporate character on customers and employees: exploring similarities and differences. Journal of the Academy of Marketing Science, v.34, n.2, p. 138-146, 2006.

DILLARD, J.; WEBER, K.; VAIL, R. The relationship between the perceived and actual effectiveness of persuasive messages: A meta-analysis with implications for formative campaign research. Journal of Communication, v. 57, n. 4, p. 613- 631, 2007.

DOREN, D. V.; CORRIGAN, H. B. Designing a marketing course with field site visits. Journal of Marketing Education, v.30, n.3, p. 189-206, 2008.

ELSBACH, K. D.; KRAMER, R. M. Members' responses to organizational identity. Administrative Science Quarterly, v. 41, n. 2, p. 442-476, 1996.

EWING, M. T.; PITT, L. F.; De BUSSY, N. M; BERTHON, P. Employment branding in the experience economy. International Journal of Advertising, v.21, n. 1, p. 3-22, 2002.

FRANKE, G. R.; HUHMANN, B. A.; MOTHERSBAUGH, D. L. Information content and consumer readership of print ads: A comparison of search and experience products. Journal of the Academy of Marketing Science, v.32, n.1, p. 20-31, 2004.

FRELING, T. H.; CROSNO, J. L.; HENARD, D. H. Brand personality appeal: conceptualization and empirical validation. Journal of the Academy of Marketing Science, v. 39, n. 3, p. 392-406, 2011.

GILLY, M. C.; WOLFINBARGER, M. Advertising's internal audience. Journal of Marketing, v.62, n.2, p. 69-88, 1998.

GOLDMAN, R.; PAPSON, S. D. Advertising in the age of hypersignification. Theory, Culture and Society, v.11, n.3, p. 23-53, 1994.

HE, H; BROWN, A. D. Organizational identity and organizational identification: A review of the literature and suggestions for future research. Group \& Organization Management, v. 38, n. 1, p. 3-35, 2013. 
HELGESEN, O.; NESSET, E. What accounts for students' loyalty? Some field study evidence. International Journal of Education, v. 21, n. 2, p. 126-143, 2007.

HEMSLEY-BROWN, J.; GOONAWARDANA, S. Brand harmonization in the international higher education market. Journal of Business Research, v.60, p. 942-948, 2007.

HENNIG-THURAU, T.; LANGER, M. F.; HANSEN, U. Modeling and managing student loyalty: An approach based on the concept of relationship quality. Journal of Service Research, v. 3, n. 4, p. 331-344, 2001.

JEVONS, C. Universities: a prime example of branding gone wrong. Journal of Product \& Brand Management, v.15, n.7, p. 466-467, 2006.

KIMPAKORN, N.; TOCQUER, G. Employees' commitment to brands in the service sector: Luxury hotel chains in Thailand. Journal of Brand Management, v.16, n.8, p. 532-544, 2009.

KLAUS, P.; MAKLAN, S. The role of brands in a service-dominated world. Journal of Brand Management, v.15, n.2, p. 115-124, 2007.

MAZZAROL, T. Critical success factors for international education marketing. International Journal of Educational Management, v.12, n.4, p. 163-175, 1998.

MICHIE, S. Pride and gratitude: how positive emotions influence the prosocial behaviors of organizational leaders. Journal of Leadership and Organizational Studies, v.15, n.4, p. 393-403, 2009.

NORIEGA, J.; BLAIR, E. Advertising to bilinguals: does the language of advertising influence the nature of thoughts? Journal of Marketing, v.72, n.5, p. 69-83, 2008.

OSMONBEKOV, T.; GREGORY, B. T.; BROWN, W.; XIE, F. T. How consumer expertise moderates the relationship between materialism and attitude toward advertising. Journal of Targeting, Measurement and Analysis for Marketing, v.17, p. 321-327, 2009.

PAPPU, R.; QUESTER, P. G.; COOKSEY, R. W. Consumer-based brand equity and countryof-origin relationships. European Journal of Marketing, v.4, n.5, p. 696-717, 2006.

PARO, V. H. A educação, a política e a administração: reflexões sobre a prática do diretor de escola. Educação e Pesquisa, São Paulo, v.36, n.3, p. 763-778, set./dez. 2010.

PETERSON, R. A.; JEONG, J. Exploring the impact of advertising and R\&D expenditures on corporate brand value and firm-level financial performance. Journal of the Academy of Marketing Science, v.38, n. 1, p. 677-690, 2010.

PUUSA, A.; KUITTINEN, M.; KUUSELA, P. Paradoxical change and construction of identity in an educational organization. Educational Management Administration \& Leadership, n. 41, v.2. p. 165-178, 2013. 
RUST, R. T. If everything is service, why is this happening now and what difference does it make?. Journal of Marketing, v.68, n.1, p. 23-24, 2004.

SCHARF, E. R. A proposta de valor e o capital humano: Práticas estratégicas de Marketing. Revista Brasileira de Gestão de Negócios, v. 14, n. 43, p. 216-233, 2012.

SCOTT, S. G.; LANE, V. R. A stakeholder approach to organizational identity. Academy of Management Review, v.25, n.1, p. 43-62, 2000.

SRIVASTAVA, R.; SHERVANI, T. A.; FAHEY, L. Marketing, business processes, and shareholder value: an organizationally embedded view of marketing activities and the discipline of marketing. Journal of Marketing, v.63, n.3, p. 168-179, 1999.

STAMP, R. The new challenge of branding buy-in. Education News, v.7, Euro RSCG Riley, 2004.

TAMBORINI, R.; BOWMAN, N. D.; EDEN, A.; GRIZZARD, M.; ORGAN, A. Defining media enjoyment as the satisfaction of intrinsic needs. Journal of Communication, v.60, p. 758-777, 2010.

TSANG, M. M.; HO, S. C.; LIANG, T. P. Consumer attitude toward mobile advertising: an empirical study. International Journal of Electronic Commerce, v.8, n.3, p. 65-78, 2004.

VERPLANKEN, B.; HOLLAND, R. W. Motivated decision making: effects of activation and self-centrality of values on choices and behavior. Journal of Personality and Social Psychology, v.82, n.3, p. 434-447, 2002.

WANG, Y.; SUN, S.; LEI, W.; TONCAR, M. Examining beliefs and attitudes toward online advertising among Chinese consumer. Journal of International Direct Marketing, v.3, n.1, p. 52-66, 2009.

WHEELER, A. R.; RICHEY, R. G.; TOKKMAN, M.; SABLYNSKI, C. J. Retaining employees for service competency: the role of corporate brand identity. Journal of Brand Management, v.14, n.1/2, p. 96-113, 2006.

WHELAN, S.; WOHLFEIL, M. Communicating brands through engagement with 'lived' experiences. Journal of Brand Management, v.13, n.4/5, p. 313-329, 2006.

YIN, R. K. Case study research: design and methods. London: Sage, 1984.

ZANCHETTA Jr., J. Circulação de textos midiáticos entre alunos de escola pública básica. Educação e Pesquisa, São Paulo, v.36, n.1, p. 297-310, jan./abr. 2010. 